\title{
COMPUTER MODELING OF PLATINUM REFORMING REACTORS
}

\author{
0. R. Momoh ${ }^{1, *}$, I. A. Mohammed-Dabo², A. S. Ahmed ${ }^{3}$ and C. S. Ajinomoh ${ }^{4}$ \\ 1,2,3,4 DePARTMENT OF CHEMICAL ENGINEERING, AHMADU BELLO UNIVERSITY, ZARIA, NIGERIA \\ E-mail addresses:1omuyar2002@yahoo.com,2iroali@mail.ru,3asahmed2007@yahoo.com, \\ ${ }^{4}$ collinajinomo@yahoo.com
}

\begin{abstract}
Usually, the reformate that is leaving any stage of the platinum reforming reactors in terms of hydrocarbon composition is assessed by laboratory analysis. The ideal composition can only be tested through theoretical means, which in most cases is avoided because of long computation time involved. This paper, instead of using a theoretical approach has considered a computer model as means of assessing the reformate composition for three-stage fixed bed reactors in platforming unit. This is done by identifying many possible hydrocarbon transformation reactions that are peculiar to the process unit, identify the operating data, mathematically model and programme their reaction rate equations using QBasic language. In this article outlet product rate of $311363.59 \mathrm{~kg} / \mathrm{hr}$ in the first, second and third reactor was obtained by modeling $275 \mathrm{~m}^{3} / \mathrm{h}$ inlet feed rate of Bayton Rouge Naphtha in the Kaduna Refinery. A manual or theoretical calculated value of $311145.22 \mathrm{~kg} / \mathrm{hr}$ of outlet product rate was obtained showing a slight deviation of $0.9 \%$. The authors are convinced that the written computer model could be applied in the process unit because it is accurate and less time consuming.
\end{abstract}

Keywords: modeling, platimun reforming, reformate, reaction rate equations, operating data

\section{INTRODUCTION}

It is well known that reforming processes were developed for the purpose of converting low octane heavy gasoline fractions (naphthas) into product with a high ignition quality, in terms of octane number [1], for blending into motor and aviation gasoline. This conversion involves subjecting the compounds in the naphtha to complex chemical reactions, at high temperature and pressure, with the aim of producing primarily aromatics and isoparaffins. In these processes by-products are also formed, mainly as a result of breakdown reactions yielding, cokes, petroleum gas (propane-butane), light hydrocarbon gases and hydrogen [2].

By process design, the various forms of reforming operations that are available in refineries worldwide are platforming, Iso Plus Houdri Forming, Rhein Forming, Power Forming, selectoforming, Ultra forming and Rex Forming. Primarily, they are all designed to perform the same function, i.e. to produce reformate that is rich in monocyclic aromatic hydrocarbons for blending with PMS and for petrochemical feed stocks. Their major differences lie in the operating conditions, nature of catalysts, product slates, feed range, etc., [2].

In this study, only platinum reforming process with fixed bed reactors would be focused upon. This is because, apart from the fact that it is the only type employed in the four refineries in Nigeria, Bhaskara Rao reported in [3] that its types are also, the most prevalent (about $90 \%$ ) in crude reforming units of the refineries worldwide.

The use of modeling tools to describe the trends and extent of products transformation in chemical engineering practices has been well studied $[4,5]$. Therefore, the aim of this work is to model platinum reforming in order to assess the reformate composition in the reactors.

\section{LITERATURE BACKGROUND}

Usually for any chemical processing, the first and foremost step is to identify as many as possible the chemistry of the process itself, represent the reactions by their rate equations reaction(s) in differential form, solve the mathematical equations, then use the results as the input data for modeling the design. Same procedure was employed here. 


\subsection{Chemistry of Platforming Reactions}

The main reactions occurring in catalytic reforming processes have been well studied [2,3]. According to [6] the followings are the major ones:

a. Dehydrogenation of naphthenic hydrocarbons yielding aromatics and hydrogen<smiles>Cc1ccccc1</smiles>

b. Dehydro-isomerisation of alkylcyclopentanes<smiles>CC1CCCC1CC1CCCCC1</smiles>

c. Dehydrocyclisation of alkanes

$$
\mathrm{C}_{7} \mathrm{H}_{16} \longrightarrow \mathrm{C}_{6} \mathrm{H}_{5} \mathrm{CH}_{3}+4 \mathrm{H}_{2}
$$

d. Cyclodehydrogenation of alkenes

$$
\mathrm{C}_{7} \mathrm{H}_{14} \longrightarrow \mathrm{C}_{6} \mathrm{H}_{5} \mathrm{CH}_{3}+3 \mathrm{H}_{2}
$$

Nelson in [7] cited that other reactions that are related to platforming are:

e. Hydrogenation of unsaturates

$\mathrm{C}_{4} \mathrm{H}_{8}+\mathrm{H}_{2} \longrightarrow \mathrm{C}_{4} \mathrm{H}_{10}$<smiles>Cc1ccccc1</smiles>

f. Hydrodesulphurisation

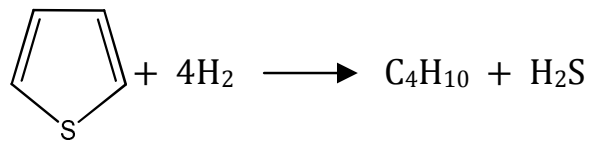

g. Denitrogenation

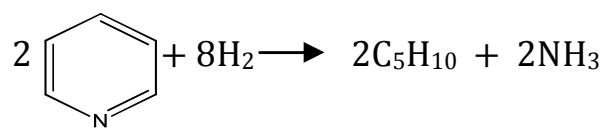

h. Deoxidation<smiles>CCOc1ccccc1</smiles>

i. Combination reactions

$2 \mathrm{RSO}_{3}+3 \mathrm{H}_{2} \longrightarrow 2 \mathrm{RH}+2 \mathrm{SO}_{2}+2 \mathrm{H}_{2} \mathrm{O}$

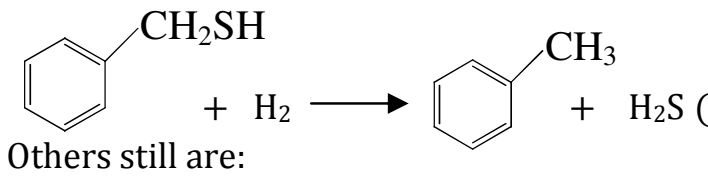

Others still are:

j. Conversion of cycloparaffins to linear paraffins

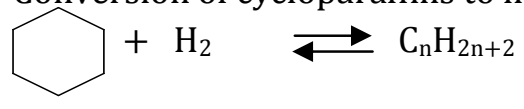

k. Hydrocracking of naphthenic hydrocarbon

$\square+\frac{n}{3} \mathrm{H}_{2} \rightarrow \frac{n}{15}\left(\mathrm{CH}_{4}+\mathrm{C}_{2} \mathrm{H}_{6}+\mathrm{C}_{3} \mathrm{H}_{8}+\mathrm{C}_{4} \mathrm{H}_{10}\right.$

$$
\left.+\mathrm{C}_{5} \mathrm{H}_{12}\right)
$$

l. Hydrocracking of paraffinic hydrocarbons

$$
\begin{aligned}
\mathrm{C}_{\mathrm{n}} \mathrm{H}_{2 \mathrm{n}+2}+ & \frac{n-3}{3} \mathrm{H}_{2} \longrightarrow \frac{n}{15}\left(\mathrm{CH}_{4}+\mathrm{C}_{2} \mathrm{H}_{6}\right. \\
& \left.+\mathrm{C}_{3} \mathrm{H}_{8}+\mathrm{C}_{4} \mathrm{H}_{10}+\mathrm{C}_{5} \mathrm{H}_{12}\right)
\end{aligned}
$$

Where $\mathrm{n}$ is the number of carbon atoms in the molecule.

However, of all the reactions listed above, those of 1 , $12,13,14$ are enough to model platforming reaction process [7] and [8] because these four reactions majorly involve the conversion of paraffins and naphthenes into branch-chained and aromatic hydrocarbons respectively. The resultant effect is the increment in octane rating and net production of hydrogen in the process [8].

According to [6] these reactions occur under the condition that the feed must be fairly free of sulphur, nitrogen and oxygen; of course, this would have been taken care of in the desulphurisation unit.

Other reactions apart from 1, 12, 13, and 14 take place concurrently and to a large extent sequentially, therefore, their reaction mechanisms and kinetics do not play significant role in the process [9].

\subsection{Catalysts and Operating Conditions}

Depending on the reforming processes type which may be semi-regenerative, or fully-regenerative or continuously regenerative, normally mono metallic or bimetallic catalysts types are employed [6] and [9]. Examples of such catalysts include platinum, platinum-rhenium, molybdenium oxide $\left(\mathrm{MoO}_{3}\right)$ supported on alumina, etc, [3, 9].

Normally reforming reactions overall, is endothermic [6] and proceed at economic rates in the temperature range of $450-530{ }^{\circ} \mathrm{C}$ to limit the catalyst performance decline rate due to coke deposition. In [7] and [8], the reaction pressure is in the range of $10-40$ bar, leading to hydrogen partial pressures in the range of 5 - 35 bar [7].

The circulation ratio of the hydrogen containing gas to the feed, as specified by [8] is about $900-1850 \mathrm{~m}^{3} / \mathrm{m}^{3}$ feed. The fractional feed cut is normally $62-180^{\circ} \mathrm{C}$, while the space velocity of the feed is between 1-3 $\mathrm{hr}^{-1}$ and the volumetric percentage composition of hydrogen in the hydrogen containing gas is $85-93 \%$ [7].

Therefore, if one can establish the reaction mechanisms of Equations 1, 12, 13 and 14 using optimal operation conditions, a mathematical model of the reaction rate equations can be programmed so that reformate composition can be obtained for process and reactor design. 


\section{PROCEDURE}

The steps that are employed in the programming are:

(a) Representation of the hydrocarbon transformation and their respective reaction kinetics.

(b) Mathematical and computer modeling of the reaction mechanisms.

\subsection{Representation of the Hydrocarbon Transformation}

As earlier mentioned, only rate reactions for reaction Equations 1,12, 13 and 14 are respectively considered in this work, which are:

I. Conversion of cycloalkanes to aromatics:

$\mathrm{C}_{\mathrm{n}} \mathrm{H}_{2 \mathrm{n}}$

$$
\mathrm{C}_{\mathrm{n}} \mathrm{H}_{2 \mathrm{n}-6}+\mathrm{H}_{2}
$$

II. Conversion of cycloparaffins to normal paraffins:

$\mathrm{C}_{\mathrm{n}} \mathrm{H}_{2 \mathrm{n}}+\mathrm{H}_{2}$

$$
\mathrm{C}_{n} \mathrm{H}_{2 \mathrm{n}+2}
$$

III. Hydrocracking of the naphthenic hydrocarbons to lighter fractions:

$$
\begin{gathered}
\mathrm{C}_{n} \mathrm{H}_{2 n}+\frac{n}{3} \mathrm{H}_{2} \\
\left.\mathrm{C}_{5} \mathrm{H}_{12}\right)
\end{gathered} \rightarrow \frac{n}{3}\left(\mathrm{CH}_{4}+\mathrm{C}_{2} \mathrm{H}_{6}+\mathrm{C}_{3} \mathrm{H}_{8}+\mathrm{C}_{4} \mathrm{H}_{10}+\right.
$$

IV. Hydrocracking of paraffinic hydrocarbons:

$$
\begin{gathered}
\mathrm{C}_{n} \mathrm{H}_{2 n+2}+\frac{n-3}{3} \mathrm{H}_{2} \rightarrow \frac{n}{15}\left(\mathrm{CH}_{4}+\mathrm{C}_{2} \mathrm{H}_{6}+\mathrm{C}_{3} \mathrm{H}_{8}+\right. \\
\left.\mathrm{C}_{4} \mathrm{H}_{10}+\mathrm{C}_{5} \mathrm{H}_{12}\right)
\end{gathered}
$$

\subsection{Reaction kinetics of the Transformation}

The four differential reaction rate equations for the four hydrocarbon transformation reactions above can be respectively represented as in [8] and they are:

$$
\begin{aligned}
-\frac{d N_{n}}{d V_{r}} & =K_{1} P_{n}-\frac{K_{1}}{K_{E 1}} P_{A}\left(P_{H}\right)^{3} \\
-\frac{d N_{n}}{d V_{r}} & =K_{2} P_{n} P_{H}-\frac{K_{2}}{K_{E 2}} \\
-\frac{d N_{n}}{d V_{r}} & =K_{3} \frac{P_{n}}{\pi} \\
-\frac{d N_{p}}{d V_{r}} & =K_{4} \frac{P_{p}}{\pi}
\end{aligned}
$$

Where, $N_{n}$ and $N_{p}$ are molar concentrations of the naphthenic and paraffinic hydrocarbons respectively in the feed;

$\mathrm{V}_{\mathrm{r}}$ - Reciprocal of the volumetric flow rate of the feed, (kg catalyst/kmol.hr); $\mathrm{K}_{1}, \mathrm{~K}_{2}, \mathrm{~K}_{3}$ and $\mathrm{K}_{4}$ - Rate constants of the four reactions respectively, kmol. /(h. Pa. kg catalyst); $\mathrm{K}_{\mathrm{E} 1}$ and $\mathrm{K}_{\mathrm{E} 2}$ - Constants of chemical equilibrium as reported [8] and can be expressed as the following:

$$
K_{E 1}=9.81^{3} * 10^{12} * e^{46.15-25600 / T}, \mathrm{~Pa}^{3}
$$

$K_{E 2}=9.81^{-1} * 10^{-3} * e^{4450 / T-7.12}, \mathrm{~Pa}^{-2}$

$T$ is expressed in degree Kelvin; $\mathrm{P}_{\mathrm{n}}, \mathrm{P}_{\mathrm{A}}, \mathrm{P}_{\mathrm{P}}$ and $\mathrm{P}_{\mathrm{H} 2}-$ are partial pressures of naphthenic, aromatic, paraffinic hydrocarbons and hydrogen in the process, $\mathrm{Pa}$.

\subsection{Mathematical and Computer Modeling of the Reaction Mechanisms}

All the expressions above were used as the basis for the mathematical modeling of the process. The next step involved the sub-routine programming of the model, which was debugged in such a manner that it could be simple and well interactive. The debugged sub-routine programmes employed include AddRec, DataScreen, Processing, Report, Reportintro and Wipe. Their meanings are explained thus:

1. AddRec is for ' $Y$ ' to save the input records and ' $N$ ' to re-enter the correct data especially if the input data was wrong.

2. DataScreen is to input data.

3. Processing which is the most important routine in the modeling is for the programming. See Appendix 1

4. Report is for the generation of the result output.

5. Reportintro serves the purpose of requesting the particular reformate record that one wishes to generate and at the same time send that record to the screen for viewing and printout if desired.

6. Wipe is to convey educative message that one is dealing with generation of result in the main menu which is boldly captioned Reformate Composition.

The following input data are required to run the programme:

Enter the Code/Record Number for the Crude $=$ Baton Rouge (See Table 1)

Feed Rate, $\mathrm{m}^{3} / \mathrm{h}=275 \mathrm{~m}^{3} / \mathrm{h}$. (as in [10])

Specific Gravity $=0.740$ (See Table 1)

Temperature in First Reactor, $\mathrm{K}=803$ (as in [8])

Temperature in Second Reactor, $\mathrm{K}=$ Depends on the output from the programme.

Temperature in Third Reactor, $\mathrm{K}=$ Depends on the output from the programme.

Pressure in First Reactor, $\mathrm{Pa}=3.43 \times 10^{6}$ (as in [7])

Pressure in Second Reactor, $\mathrm{Pa}=$ Depends on the output from the programme.

Pressure in Third Reactor, $\mathrm{Pa}=$ Depends on the output from the programme.

Space Velocity, $1 / \mathrm{h}=1.5 \quad$ (as in [7])

Hydrogen - Containing Circulation Ratio, $\mathrm{m}^{3} / \mathrm{m}^{3}=$ 1500 (as in [2])

Paraffinic content, wt. \%. (See Table 1 for Baton Rouge) 
Naphthenic content, wt. \% (See Table 1 for Baton Rouge)

Aromatic content, wt. \% (See Table 1 for Baton Rouge)

ASTM distillation Temp of $50 \%$ feed, K (See Table 1 for Baton Rouge)

Bulk Density of Alumnoplatinium Catalyst $=600$ $\mathrm{kg} / \mathrm{m}^{3}$ (as in [8]).

Meanwhile Table 1 displays the physical properties of naphthas that were obtained from five different crudes. These properties partly act as the source of input data as requested above

Table 1: Properties of Naphtha Obtained from Five Crudes

\begin{tabular}{llllll}
\hline $\begin{array}{l}\text { Crude type } \rightarrow \\
\text { Properties } \downarrow\end{array}$ & $\begin{array}{c}\text { Baton } \\
\text { Rouge }\end{array}$ & $\begin{array}{c}\text { Arabian } \\
\text { Light }\end{array}$ & $\begin{array}{c}\text { Arabian } \\
\text { Heavy }\end{array}$ & Pona & Baytown \\
\hline $\begin{array}{l}\text { Paraffin, } \\
\text { vol.\% }\end{array}$ & 53 & 66.3 & 70.3 & 50.4 & 38.1 \\
$\begin{array}{l}\text { Naphthenes, } \\
\text { vol. \% }\end{array}$ & 39.9 & 20.0 & 21.4 & 39.2 & 42.6 \\
$\begin{array}{l}\text { Aromatics, } \\
\text { vol. \% }\end{array}$ & 10.1 & 13.7 & 8.3 & 10.4 & 19.3 \\
$\begin{array}{l}\text { Specific } \\
\text { gravity }\end{array}$ & 0.740 & 0.744 & 0.737 & 0.745 & 0.767 \\
\hline ASTM Distillation, ${ }^{\circ} \mathrm{C}$ & & & & \\
\hline IBP & 85 & 100 & 100 & 70 & 99 \\
$\begin{array}{l}10 \% \\
50 \%\end{array}$ & 92 & 105 & 107 & 79 & 115 \\
$90 \%$ & 102 & 125 & 124 & 110 & 134 \\
FBP & 128 & 145 & 146 & 142 & 157 \\
\hline
\end{tabular}

Source: Reforming Unit, Kaduna Refinery and Petrochemical Company, KRPC (2002)

Table 2: Summary of the Result Obtained for Reforming Baton Rouge Naphtha Using the QBasic programming

\begin{tabular}{c|c|c}
\hline \multicolumn{3}{|c}{ The first reactor , kg/h } \\
\hline & Inflow & Outflow \\
\hline Aromatics & 20207.5492 & 86971.7358 \\
Naphthenes & 75111.8545 & 8639.4837 \\
Paraffins & 108180.6032 & 96885.1518 \\
Hydrogen & 107863.5796 & 118867.2152 \\
\hline Total & 311363.5865 & 311363.5865 \\
\hline \multicolumn{3}{|c}{ The second reactor , kg/h } \\
\hline Aromatics & 86971.7358 & 90682.7894 \\
Naphthenes & 8639.4837 & 0 \\
Paraffins & 96885.1518 & 87633.1051 \\
Hydrogen & 118867.2152 & 133047.6920 \\
\hline Total & 311363.5865 & 311363.5865 \\
\hline \multicolumn{3}{|c}{ The third reactor , kg/h } \\
\hline Aromatics & 90682.7894 & 90682.7894 \\
Paraffins & 87633.1051 & 88515.8367 \\
Hydrogen & 133047.6920 & 132164.9604 \\
\hline Total & 311363.5865 & 311363.5865 \\
\hline
\end{tabular}

\section{RESULTS}

Baton Rouge naphtha was employed for the mathematical model as well as for the

Programming. Though oversimplified the result or material balance obtained as in [8] and [11] is displayed in Table 2:

\subsection{Discussion}

The result obtained above is based on the feed rate of the reactor at $275 \mathrm{~m}^{3} / \mathrm{h}$, which is in conformity with [10].

Suffice to say that the model was equally employed for other naphthas that were obtained from the other sources and results obtained were of the same pattern as in Table 2.

With regards to the values obtained in Table 2 , it can be observed that the naphthenic content of the naphtha drastically reduced in the first reactor. This is expected because aromatization of the naphthenes is highly favored in the first reactor [8], [12] and [13]. Meanwhile, the hydrogen produced has gone ahead to enrich the hydrogen -containing gas that was initially charged into the reactor as seen in the outflow of hydrogen in the first reactor. In the second reactor, hydrogen again has increased through the aromatization of the remaining naphthenes. But in the last reactor the hydrogen content has reduced because part of it has been utilized for the hydrocracking of paraffinic contents of the feed. The paraffinic content initially reduced in the first and second reactor due to hydrocracking but again picked up in the third reactor because this process enhances production of paraffinic hydrocarbon gases.

\section{CONCLUSION}

The use of the modeling tools to describe the trends in the platinum reforming of naphtha has been demonstrated. The authors are convinced that this computer modeling and simulation of platinum reforming process is very efficient and the data obtained are reliable. As a result it can be used to assess and monitor the reformate composition as it leaves one stage of the reactors to another. In essence, the data obtained from it can act as a check to the information obtained from theoretical approach and laboratory.

The model is equally highly recommended for prototype and industrial design of platinum reforming reactors.

\section{REFERENCES}

[1] Peer, R.L. Bennett, R.W. Felch, D.E. and Kabza, R.G. (1990): PLATFORMING. Leading Octane Technology into The 1990's. UOP Partnership For The 1990's, pp.1-2.

[2] Erikh, V.N. Rasina, M.G. and Rudin, M.G. (1988): The Chemistry and Technology of Petroleum and Gas, Moscow. pp.234-252. 
[3] Bhaskara Rao, B.K. (2007): Modern Petroleum Refining Processes, $5^{\text {th }}$ edition, published by Oxford and IBH Publishing, Co.PVT. Ltd, New Delhi, pp.344360.

[4] N.A. Amenaghawon, E.I. Osagie, S.O. Osemwengie, S.E. Ogbeide and C.O. Okieimen (2013): Modeling and Simulation of the Batch Hydrolysis of Acetic Anhydride to Produce Acetic Acid. Nigerian Journal of Technology, Vol. 32, No. 3, pp 386- 392.

[5] M.C. Ndukwu, S.I. Manuwa, O.J. Olukunle and I.B. Oluwalana (2013): Mathematical Model for Direct Evaporative Space Cooling Systems. Nigerian Journal of Technology, Vol. 32, No. 3, pp. 403- 409.

[6] Baxendell, P. (1983): The Petroleum Handbook, 6 th $^{\text {th }}$ Edition, compiled by staff of the Royal Dutch/Shell Group of Companies Elsevier, pp.268-276.

[7] Nelson W.L. (1969): Petroleum Refinery Engineering, Catalytic Cracking and Reforming, $4^{\text {th }}$ Edition, McGraw-Hill Book Company, New York, USA. pp. 810-818.

[8] Kuznyesov, A.A., Kagermanov, S.M. and Sudakov, E.N., (1974): Calculations of Unit Operations in Petroleum Industries. Leningrad: Khimia. pp.251-288.

[9] Speight, J. G. (1999): The Chemistry and Technology of Petroleum, $3^{\text {rd }}$ edition, New York Based, pp.684693.

[10] Reforming Unit Record, (2002). Kaduna Refinery and Petrochemicals Company, KRPC Ltd, Kaduna State, Nigeria

[11] Coulson, J.M., Richardson, J.F. and Peacock, D.G. (1979): Chemical Engineering Vol.3, Chemical Reactor Design; Biochemical Reaction Engineering including computational techniques and control. $2^{\text {nd }}$ ed. (SI units). Pergamon Press. Oxford. Britain, pp.12-14.

[12] Van Dyke K. (1997): Fundamentals of Petroleum; Catalytic Reforming, $4^{\text {th }}$ edition. Published by Petroleum Extension Services, Continuing \& Extended Education. The University of Texas at Austin; Austin, Texas, USA, p. 281.

[13] http://chemcases.com/converter/converter-f.htm (1998), accessed March 15, 2006.

\section{Appendix 1}

SUB Processing

$\mathrm{Mf}=105$

$\mathrm{n}=7.4538$

Hydro $=.86$

Methane $=.04$

Ethane $=.05$

Propane $=.03$

Buthane $=.01$

Penthane $=.01$

$\mathrm{Ma}=\left(14^{*} \mathrm{n}\right)-6$
$\mathrm{Mn}=14 * \mathrm{n}$

$\mathrm{Mp}=\left(14^{*} \mathrm{n}\right)+2$

$\mathrm{Ya}=(\mathrm{Mf} / \mathrm{Ma}) *$ FileRec.AW

$\mathrm{Yn}=(\mathrm{Mf} / \mathrm{Mn}) *$ FileRec.NW

$\mathrm{Yp}=(\mathrm{Mf} / \mathrm{Mp}) *$ FileRec.PW

$\mathrm{RRC} 2=2.398 * 10^{\wedge}-15$

$\mathrm{RRC} 1=.0000003145 \#$

$\mathrm{RRC} 3=.1$

RRC4 = RRC3

SecRRC1 $=290.5 * 10^{\wedge}(-9)$

SecRRC4 $=.0714$

ThirdRRC4 $=.06178$

GF $=$ FileRec.SPgravity * $1000 *$ FileRec.FeedRate

TotalMoleOfFeed $=\mathrm{GF} / \mathrm{Mf}$

TMMOfAromatic $=$ TotalMoleOfFeed $*$ Ya

TMMOfParafin $=$ TotalMoleOfFeed ${ }^{*}$ Yp

TMMOfNaphthene $=$ TotalMoleOfFeed $*$ Yn

TotalMolarMass $=$ TMMOfAromatic + TMMOfParafin + TMMOfNaphthene

$\mathrm{GH}=(\mathrm{GF} *$ FileRec.HCCR $) /(1000 *$ FileRec.SPgravity $)$

$\mathrm{NH}=\mathrm{GH} / 22.4$

MMHydrogen $=2$

MMMethane $=16$

MMEthane $=30$

MMPropane $=44$

MMButhane $=58$

MMPentane $=72$

MRHydrogen $=$ Hydro $*$ MMHydrogen

MRMethane $=$ Methane $*$ MMMethane

MREthane $=$ Ethane ${ }^{*}$ MMEthane

MRPropane $=$ Propane $*$ MMPropane

MRButhane $=$ Buthane $*$ MMButhane

MRPentane $=$ Penthane $*$ MMPentane

TotalMR $=$ MRHydrogen + MRMethane + MREthane +

MRPropane + MRButhane + MRPentane

NHHydro $=\mathrm{NH}^{*}$ Hydro

$\mathrm{NHHydro}=\mathrm{NH}^{*}$ Hydro

NHMethane $=\mathrm{NH}^{*}$ Methane

NHEthane $=\mathrm{NH}^{*}$ Ethane

NHPropane $=\mathrm{NH}^{*}$ Propane

NHButhane $=\mathrm{NH}^{*}$ Buthane

NHPentane $=\mathrm{NH}^{*}$ Penthane

TotalNHi $=$ NHHydro + NHMethane + NHEthane +

NHPropane + NHButhane + NHPentane

KMolePerHour $=$ TotalNHi - NHHydro

TotalAmount $=$ TMMOfAromatic + TMMOfParafin +

TMMOfNaphthene + NHHydro + KMolePerHour

MfAromatic $=$ TMMOfAromatic $/$ TotalAmount

MfNaphthene $=$ TMMOfNaphthene $/$ TotalAmount

MfParafin = TMMOfParafin $/$ TotalAmount

MfHydro = NHHydro $/$ TotalAmount

MfKMolePerHour = KMolePerHour $/$ TotalAmount

PpOfAromatic $=$ FileRec.PFR $*$ MfAromatic

PpOfNaphthene $=$ FileRec.PFR $*$ MfNaphthene 
PpOfParafin $=$ FileRec.PFR $*$ MfParafin

PpOfHydro $=$ FileRec.PFR $*$ MfHydro

PpOfKmolePerHour $=$ FileRec.PFR $*$ MfKMolePerHour

TotalPp $=$ PpOfAromatic + PpOfNaphthene +

PpOfParafin + PpOfHydro + PpOfKmolePerHour

$\mathrm{VC}=\mathrm{GF} /($ FileRec.SPgravity $* 1000 *$ FileRec.SV)

$\mathrm{GC}=\mathrm{VC} *$ FileRec.BDAC

VolOfFirstReactor $=\mathrm{VC} / 7$

VolOfSecondReactor $=(2 *$ VC $) / 7$

VolOfThirdReactor $=(4 *$ VC $) / 7$

\section{REM FIRST REACTOR COMPUTATION}

$\mathrm{KP} 1=14.866 * 10^{\wedge} 20$

$\mathrm{Nn}=(\mathrm{RRC} 1 *$ PpOfNaphthene $)-(\mathrm{RRC} 1 / \mathrm{KP} 1) *$

PpOfAromatic * PpHydro

GCAromatic $=$ FileRec.BDAC $*$ VolOfFirstReactor

VRi = GCAromatic / TotalMolarMass

REM now computes the total amount of naphthene

converted into Aromatic

$\mathrm{Nni}=\mathrm{Nn} * \mathrm{VRi}$

REM to compute the remaining amount of Naphthene left in the First Reactor

NleftinFirstReactor $=(\mathrm{Yn}-\mathrm{Nni}) *$ TotalMolarMass

REM to compute the amount of naphthene converted to aromatic in the first reactor

NconvertedtoA = TMMOfNaphthene -

NleftinFirstReactor

REM using reaction rate constant 2 for the first reactor

$\mathrm{Kp} 2=2.103^{*} 10^{\wedge}(-6)$

DNni2 $=($ RRC2 $*$ PpOfNaphthene $*$ PpOfHydro $)-($ RRC2

* PpOfParafin) / Kp2

$\mathrm{Nni} 2=\mathrm{DNni} 2 * \mathrm{VRi}$

IncrementInNNi2 $=((\mathrm{Yn}-\mathrm{Nni})+\mathrm{Nni} 2) *$

TotalMolarMass

MQNaphtheneToParafin = IncrementInNNi2 -

NleftinFirstReactor

REM computing for reaction rate constant 3 for the first reactor

DNni3 $=$ RRC3 $*$ PpOfNaphthene $/$ FileRec.PFR

$\mathrm{Nni} 3=\mathrm{DNni} 3 * \mathrm{VRi}$

MQofNaphtheneRem $=(($ Yn $-\mathrm{Nni})+(\mathrm{Nni} 2-\mathrm{Nni} 3)) *$

TotalMolarMass

MQNHydrocracking = IncrementInNNi2 -

MQofNaphtheneRem

REM computing for reaction rate constant 4 for the first reactor

DNp1 $=$ RRC4 $*$ PpOfParafin / FileRec.PFR

$\mathrm{Np} 1=\mathrm{DNp} 1 * \mathrm{VRi}$

Np14 $=($ Yp - Np1 $) *$ TotalMolarMass

Npgi $=$ TMMOfParafin - Np14

AmtOfGasInFirstReactor $=($ MQNHydrocracking + Npgi) * n / 15

ProductAromatic $=$ TMMOfAromatic + NconvertedtoA
ProductNaphthene $=($ TMMOfNaphthene -

NconvertedtoA) + (MQNaphtheneToParafin -

MQNHydrocracking)

ProductParafin = TMMOfParafin -

MQNaphtheneToParafin - Npgi

TotProduct $=$ ProductAromatic + ProductNaphthene +

ProductParafin

Musty $=$ NconvertedtoA $* 3$

Adams $=($ MQNHydrocracking $* n) / 3$

Onimisi $=(\operatorname{Npgi} *(\mathrm{n}-3)) / 3$

HCG $=$ NHHydro + Musty + MQNaphtheneToParafin -

Adams - Onimisi

MCG $=$ NHMethane + AmtOfGasInFirstReactor

ECG $=$ NHEthane + AmtOfGasInFirstReactor

PCG $=$ NHPropane + AmtOfGasInFirstReactor

BCG $=$ NHButhane + AmtOfGasInFirstReactor

PentCG $=$ NHPentane + AmtOfGasInFirstReactor

TotCG = HCG + MCG + ECG + PCG + BCG + PentCG

Gtotal $=$ TotCG + TotProduct

HCGMF = HCG $/$ TotCG

MCGMF $=$ MCG $/$ TotCG

$\mathrm{ECGMF}=\mathrm{ECG} / \mathrm{TotCG}$

PCGMF $=$ PCG $/$ TotCG

BCGMF $=$ BCG $/$ TotCG

PentCGMF $=$ PentCG $/$ TotCG

MYHydro $=$ HCGMF $^{*}$ MMHydrogen

MYMethane $=$ MCGMF $*$ MMMethane

MYEthane $=$ ECGMF $*$ MMEthane

MYPropane $=$ PCGMF $*$ MMPropane

MYButhane $=$ BCGMF $*$ MMButhane

MYPenthane $=$ PentCGMF $*$ MMPentane

TotMY = MYHydro + MYMethane + MYEthane +

MYPropane + MYButhane + MYPentane

AmtOfHydroRichGas $=$ TotCG $*$ TotMY

\section{REM THIS SHOULD BE MY INLET OUTPUT-}

FileRec.GiAromatic $=$ TMMOfAromatic $*$ Ma

FileRec.GiNaphthene $=$ TMMOfNaphthene ${ }^{*} \mathrm{Mn}$

FileRec.GiParafin $=$ TMMOfParafin ${ }^{*} \mathrm{Mp}$

GiHydro $=$ NHHydro $*$ TotalMR $/ .86$

FileRec.GiHydroParaf $=($ KMolePerHour + NHHydro $) *$

TotMY

TotalGinlet $=$ FileRec.GiAromatic +

FileRec.GiNaphthene + FileRec.GiParafin + GiHydro

TotOrganicCG = MCG + ECG + PCG + BCG + PentCG

FileRec.GoHydroParaf $=(\mathrm{HCG}+$ TotOrganicCG $) *$

TotMY

QtyHCLeavingReactor $=$ TotalGinlet -

FileRec.GoHydroParaf

$\mathrm{ZZ}=6^{*}$ ProductAromatic

$\mathrm{KKZ}=2 *$ ProductParafin

$\mathrm{XXX}=$ ProductAromatic + ProductNaphthene +

ProductParafin 
FirstN $=((Q$ tyHCLeavingReactor $+\mathrm{ZZ})-\mathrm{KKZ}) /(14 *$

XXX)

$$
\begin{aligned}
& \text { FirstMa }=\left(14^{*} \text { FirstN }\right)-6 \\
& \text { FirstMn }=14^{*} \text { FirstN } \\
& \text { FirstMp }=\left(14^{*} \text { FirstN }\right)+2
\end{aligned}
$$

\section{REM THIS SHOULD BE MY OUTLET OUTPUT}

FileRec.GoAromatic $=$ ProductAromatic $*$ FirstMa FileRec.GoNaphthene $=$ ProductNaphthene ${ }^{*}$ FirstMn FileRec.GoParafin $=$ ProductParafin ${ }^{*}$ FirstMp 'chy $=$ HCG $*$ TotMY / HCGMF

TotalMoleOutlet $=$ ProductAromatic +

ProductNaphthene + ProductParafin + HCG + TotOrganicCG

MFOutletAromatic $=$ ProductAromatic $/$

TotalMoleOutlet

MFOutletNaphthene = ProductNaphthene /

TotalMoleOutlet

MFOutletParafin = ProductParafin $/$ TotalMoleOutlet MFOutletHydro = HCG / TotalMoleOutlet

MFOutletOrganic $=$ TotOrganic $/$ TotalMoleOutlet

TotANP = FileRec.GoAromatic + FileRec.GoNaphthene

+ FileRec.GoParafin

TotGoutlet $=$ TotANP + FileRec.GoHydroParaf

PFR2 $=3130000$

TFR2 $=793$

PpAromatic2 $=$ FileRec.PFR2 $*$ MFOutletAromatic PpNaphthene $2=$ FileRec.PFR2 $*$ MFOutletNaphthene PpParafin2 $=$ FileRec.PFR2 $*$ MFOutletParafin PpHydro2 $=$ FileRec.PFR2 $*$ MFOutletHydro PpOrganic $=$ FileRec.PFR $2 *$ MFOutletOrganic REM REACTION RATE CONSTANT FOR SECOND REACTOR

SecRxtMFAroma $=$ ProductAromatic $/$ TotProduct SecRxtMFNaph $=$ ProductNaphthene $/$ TotProduct SecRxtMFParaf $=$ ProductParafin $/$ TotProduct KPSecRxtor $=9.94 * 10^{\wedge} 20$

HPower $=$ PpHydro $2^{\wedge} 2$

DNn2 $=($ SecRRC1 $*$ PpNaphthene $)-(($ SecRRC1 $*$

PpAromatic * HPower) / KPSecRxtor)

GC2 = FileRec.BDAC $*$ VolOfSecondReactor

$\mathrm{VR} 2=\mathrm{GC} 2 /$ TotProduct

NnSecondRxtor $=\mathrm{DNn} 2 * \mathrm{VR} 2$

NewVolNaph $=$ SecRxtMFNaph $/$ DNn2

NCatalystQty $=$ NewVolNaph $*$ TotProduct

RedCat $=$ GC2 - NCatalystQty

NnSecondRxtor $=$ SecRxtMFNaph

FileRec.NRemAromatize $=($ NnSecondRxtor -

SecRxtMFNaph) * TotProduct

REM USING REACTION RATE CONSTANT 4 FOR THE

FIRST REACTOR

DNp2 $=($ SecRRC4 $*$ PpParafin2 $) /$ FileRec.PFR2

NpSecondReactor $=$ DNp2 $*$ NewVolNaph
NPHydrocracking $=($ SecRxtMFParaf -

NpSecondReactor) * TotProduct

AmtPconToGas $=$ ProductParafin - NPHydrocracking

SecRxtOutletAroma $=$ ProductAromatic +

ProductNaphthene

SecRxtOutletParaf $=$ ProductParafin - AmtPconToGas

TotSecRxtOutlet $=$ SecRxtOutletAroma +

SecRxtOutletParaf

AmtGasLeavSecRxt $=$ AmtPconToGas * $(\mathrm{n} / 15)$

HCG2 $=$ HCG $+($ ProductNaphthene $* 3)-$

AmtPconToGas * (n - 3) / 3

MCG2 = MCG + AmtGasLeavSecRxt

$\mathrm{ECG} 2=\mathrm{ECG}+$ AmtGasLeavSecRxt

PCG2 = PCG + AmtGasLeavSecRxt

BCG2 = BCG + AmtGasLeavSecRxt

PentCG = PentCG + AmtGasLeavSecRxt

TotOrgCG2 = MCG2 + ECG2 + PCG2 + BCG2 + PentCG2

TotCG2 = HCG2 + TotOrgCG2

MFYG2Hydro = HCG2 $/$ TotCG2

MFYG2Methane $=$ MCG2 $/$ TotCG2

MFYG2Ethane $=$ ECG2 $/$ TotCG2

MFYG2Propane $=$ PCG2 $/$ TotCG2

MFYG2Buthane $=$ BCG2 $/$ TotCG2

MFYG2Pentane $=$ PentCG2 $/$ TotCG2

MyG2Hydro = MMHydrogen * MFYG2Hydro

MyG2Methane $=$ MMMethane $*$ MFYG2Methane

MyG2Ethane $=$ MMEthane $*$ MFYG2Ethane

MyG2Propane $=$ MMPropane $*$ MFYG2Propane

MyG2Buthane $=$ MMButhane ${ }^{*}$ MFYG2Buthane

MyG2Pentane $=$ MMPentane $*$ MFYG2Pntane

TotMYG2 = MyG2Hydro + MyG2Methane +

MyG2Ethane + MyG2Propane + MyG2Buthane + MyG2Pentane

HCRGIncreasedBy = HCG2 + TotCG2 $*$ TotMYG2

HCLeavSecRxt $=$ TotGoutlet - HCRGIncreasedBy

MFOutletSecRxtA = SecRxtOutletAroma / TotCG2

MFOutletSecRxtP = SecRxtOutletParaf / TotCG2

MFOutletSecHydro = HCG2 / TotCG2

MFTotOrganicCG = TotOrganicCG / TotGoutlet

$\mathrm{nSec}=($ HCLeavSecRxt $-(6 *$ SecRxtOutletAroma $)-(2 *$ SecRxtOutletParaf)) / (14* (SecRxtOutletAroma + SecRxtOutletParaf))

SecMa $=(14 * n$ Sec $)-6$

$\operatorname{SecMp}=(14 * \mathrm{nSec})+2$

FileRec.MFR2Aromatic $=$ SecRxtOutletAroma $*$ SecMa

FileRec.MFR2Parafin $=$ SecRxtOutletParaf $*$ SecMp

FileRec.MFR2Hydroparaf $=($ HCG2 + TotOrgCG2 $) *$

TotMYG2

TotMFR2 = FileRec.MFR2Aromatic +

FileRec.MFR2Parafin + FileRec.MFR2Hydroparaf

$\mathrm{PFR} 3=3 * 10^{\wedge} 6$

TotThirdRxt $=$ SecRxtOutletAroma + SecRxtOutletParaf 
MFThirdRxtAroma = SecRxtOutletAroma $/$ TotThirdRxt MFThirdRxtParaf $=$ SecRxtOutletParaf $/$ TotThirdRxt ThirdRxtPpAroma = MFOutletSecRxtA * FileRec.PFR3 ThirdRxtPpParaf $=$ MFOutletSecRxtP ${ }^{*}$ FileRec.PFR3 ThirdRxtPpHydro = MFOutletSecHydro * FileRec.PFR3 ThirdRxtPpOrganicCG = MFTotOrganicCG *

FileRec.PFR3

TotThirdPp $=$ ThirdRxtPpParaf + ThirdRxtPpAroma + ThirdRxtPpHydro + ThirdRxtPpOrganicCG DNn3 $=$ ThirdRRC4 $*$ ThirdRxtPpParaf / FileRec.PFR3 Gc3Alone $=$ FileRec.BDAC $*$ VolOfThirdReactor Gc3 = Gc3Alone + RedCat

VR3 = Gc3 / TotThirdRxt

ThirdRxtNn3 = DNn3 $*$ VR3

NPRemHydrocrack $=($ MFThirdRxtParaf - ThirdRxtNn3 $)$

* TotThirdRxt

NPgThirdRxt $=$ SecRxtOutletParaf - NPRemHydrocrack ThirdOutletAroma = SecRxtOutletAroma

ThirdOutletParaf = SecRxtOutletParaf - NPgThirdRxt TotOutletPA $=$ ThirdOutletAroma + ThirdOutletParaf AmtGhirdRxt $=($ NPgThirdRxt $*$ nSec $) / 15$

HCG3 $=$ HCG2 $+($ NPgThirdRxt $*((n S e c-3) / 3))$

MCG3 = MCG2 + AmtGhirdRxt

$\mathrm{ECG} 3=\mathrm{ECG} 2+$ AmtGhirdRxt

PCG3 = PCG2 + AmtGhirdRxt

BCG3 = BCG2 + AmtGhirdRxt

PentCG3 = PentCG2 + AmtGhirdRxt

TotCG3 = HCG3 + MCG3 + ECG3 + PCG3 + BCG3 +

PentCG3

MFYG3Hydro = HCG3 $/$ TotCG3

MFYG3Methane $=$ MCG3 $/$ TotCG3

MFYG3Ethane = ECG3 $/$ TotCG3

MFYG3Propane $=$ PCG3 $/$ TotCG3

MFYG3Buthane $=$ BCG3 $/$ TotCG3

MFYG3Pentane $=$ PentCG3 $/$ TotCG3

MyG3Hydro = MMHydrogen * MFYG3Hydro
MyG3Methane $=$ MMMethane $*$ MFYG3Methane MyG3Ethane $=$ MMEthane $*$ MFYG3Ethane MyG3Propane $=$ MMPropane $*$ MFYG3Propane MyG3Buthane $=$ MMButhane $*$ MFYG3Buthane MyG3Pentane $=$ MMPentane $*$ MFYG3Pntane TotMYG3 = MyG3Hydro + MyG3Methane + MyG3Ethane + MyG3Propane + MyG3Buthane + MyG3Pentane TotOrganicCG3 = MCG3 + ECG3 + PCG3 + BCG3 + PentCG3

ThirdHCRGIncBy $=($ HCG3 + TotOrganicCG3 $) *$ TotMYG3

QtyHCleavThirdRxt $=$ TotMFR2 - ThirdHCRGIncBy

MFOutletThirdRxtA $=$ ThirdOutletAroma

MFOutletThirdRxtP $=$ ThirdOutletParaf

MFThirdOutletHydro = HCG3

MFThirdTotOrgCG = TotOrganicCG3

TotThirdOutlet $=$ MFOutletThirdRxtA +

MFOutletThirdRxtP + MFThirdOutletHydro +

MFThirdTotOrgCG

TotDen $=$ MFOutletThirdRxtA + MFOutletThirdRxtP

nThird $=\left(\right.$ QtyHCleavThirdRxt $+\left(6^{*}\right.$

MFOutletThirdRxtA) - $(2 *$ MFOutletThirdRxtP) $) /(14 *$

TotDen)

ThirdMa $=(14 *$ nThird $)-6$

ThirdMp $=(14 *$ nThird $)+2$

FileRec.MFR3Aromatic $=$ SecRxtOutletAroma $*$ ThirdMa

FileRec.MFR3Parafin $=$ SecRxtOutletParaf $*$ ThirdMp

FileRec.MFR3HydroParaf $=($ HCG2 + TotOrganicCG3 $) *$

TotMYG3

TotMFR3 = FileRec.MFR3Aromatic +

FileRec.MFR3Parafin + FileRec.MFR3HydroParaf

ThirdMa $=14 *($ nThird $)-6$

ThirdMp $=14 *($ nThird $)+2$

END SUB 